OPEN

SUBJECT AREAS:

MACROAUTOPHAGY

ENDOCRINE REPRODUCTIVE

DISORDERS

Received

13 November 2014

Accepted

10 February 2015

Published

9 March 2015

Correspondence and requests for materials should be addressed to H.-L.D. (dr_dhl@126.

com) or X.-N.K. |xiaonankang@126.

com)

\section{Testosterone regulates the autophagic clearance of androgen binding protein in rat Sertoli cells}

\author{
Yi Ma ${ }^{1,2}$, Hao-Zheng Yang ${ }^{3}$, Long-Mei Xu3 ${ }^{3}$ Yi-Ran Huang ${ }^{2}$, Hui-Li Dai ${ }^{1} \&$ Xiao-Nan Kang ${ }^{1}$
}

\begin{abstract}
'Department of Biobank, Renji Hospital, School of Medicine, Shanghai JiaoTong University, Building 1, 1630 DongFang Road, Shanghai, 200127, China, ${ }^{2}$ Department of Urology, Renji Hospital, School of Medicine, Shanghai JiaoTong University, Building 7, 1630 DongFang Road, Shanghai, 200127, China, ${ }^{3}$ Department of Central Laboratory, Renji Hospital, School of Medicine, Shanghai JiaoTong University, Building 5, 1630 DongFang Road, Shanghai, 200127, China.
\end{abstract}

Dysregulation of androgen-binding protein (ABP) is associated with a number of endocrine and andrology diseases. However, the ABP metabolism in Sertoli cells is largely unknown. We report that autophagy degrades ABP in rat Sertoli cells, and the autophagic clearance of ABP is regulated by testosterone, which prolongs the ABP biological half-life by inhibiting autophagy. Further studies identified that the autophagic clearance of ABP might be selectively regulated by testosterone, independent of stress (hypoxia)-induced autophagic degradation. These data demonstrate that testosterone up-regulates ABP expression at least partially by suppressing the autophagic degradation. We report a novel finding with respect to the mechanisms by which ABP is cleared, and by which the process is regulated in Sertoli cells.

S ex hormone-binding globulin (SHBG) is a glycoprotein that is mostly produced by the liver, testes, brain, and placenta. Testes-produced SHBG is also called androgen-binding protein (ABP). ABP specifically binds to testosterone or dihydrotestosterone, making them less lipophilic and more concentrated in the seminiferous tubules. High levels of ABP are essential for maintaining the microenvironment as well as enable spermatogenesis in the seminiferous tubules and sperm maturation in the epididymis. ABP can promote germ cell differentiation, and it has been reported that ABP up-regulates the expression of transition protein 1 (TP1), which is involved in nuclear chromatin condensation in rodent spermatids ${ }^{1}$. ABP can also directly up-regulate the expression of aromatase in germ cells and oestrogen receptor beta ${ }^{2}$. In humans, an alternative ABP transcript accumulates in germ cells in a highly regulated manner throughout the spermatogenic cycle ${ }^{3}$, and its expression is positively correlated with sperm motility ${ }^{4}$. It has been reported that human ABP transcription is controlled by several germ cell-enriched transcription factors, including SPZ1 and CREB/CREM ${ }^{5}$. When ABP migrates together with immature sperm to the caput epididymis, it promotes the access of testosterone to the immature sperm and promotes sperm maturation ${ }^{6}$. ABP also aids in the transport of testosterone, dihydrotestosterone and oestrogens throughout the body, which continue to play a role in the target organs. The affinity of ABP is four to five orders of magnitude greater than that of albumin; therefore, it enhances the solubility of those lipophilic molecules and prolongs their biological half-life.

The synthesis and secretion of ABP is regulated by follicle-stimulating hormone (FSH), testosterone, oestradiol, and other factors ${ }^{7-9}$. Aberrant control of ABP production has been found in several endocrine and andrology disorders ${ }^{10,11}$. Varicocele-induced dysregulation of ABP may be a parameter of impaired reproduction function ${ }^{10}$, and significantly lower ABP secretion in non-obstructed azoospermic $(\mathrm{NOA})$ patients compared with obstructed azoospermic (OA) patients may indicate a defect in ABP synthesis in NOA patients ${ }^{11}$. However, it is still not clear how the process of ABP metabolism works in Sertoli cells or how the process is regulated.

Autophagy is active in Sertoli cells ${ }^{12,13}$. Autophagy is a lysosomal degradation pathway that eliminates defective organelles and proteins from the cell. Classic macroautophagy is initiated from an isolated membrane (phagophore), which is followed by the formation of a double-membrane autophagosome that then delivers the engulfed proteins and fuses with lysosomes for degradation. MTOR signalling and ATG family members strictly regulate autophagy. In Sertoli cells, autophagy can serve as a cell survival pathway or cooperate with apoptosis to promote cell death ${ }^{14-16}$, in addition to degrading ingested cell-derived substrates ${ }^{13}$. However, the function of autophagy in Sertoli cells is largely unknown.

In this study, we have revealed that autophagy clears ABP in rat Sertoli cells, and this process appears to be selectively regulated by testosterone and independent of stress (hypoxia)-induced autophagic degradation. 


\section{Results}

Autophagy regulates the ABP expression level in rat Sertoli cells. Primary Sertoli cells were isolated from 18-22-day-old rats, and the cells isolated at this age had almost negligible somatic (i.e., Leydig cells) cell contamination ${ }^{17}$. We used oil red O and bodipy 493/503 to detect lipid droplets in Sertoli cells and used alkaline phosphatase staining to label germ cells. Lipid droplets were found in nearly all cells, but only a few cells were alkaline phosphatase positive (Fig. 1A), indicating a very high purity of the Sertoli cells used in this study.

To investigate the role of autophagy in the regulation of ABP expression, we treated primary rat Sertoli cells with chloroquine (CQ) or rapamycin, and found that CQ inhibited autophagy, while rapamycin enhanced autophagy, which is indicated by the LC3II/ LC3I ratio (Fig. 1B). We then detected the ABP expression levels with both treatments, and we found that the suppression of autophagy promoted ABP expression, while stimulation of autophagy reduced the ABP expression (Fig. 1B, 1C, 1D). In addition, both treatments changed the ABP levels that Sertoli cells secreted to the supernatant (Fig. 1E). To further verify the results, we performed gene knockdown experiments with small interfering RNA (siRNA). The results demonstrated that inhibition of autophagy by ATG7 siRNA promoted ABP expression, while stimulation of autophagy by MTOR siRNA reduced ABP expression (Fig. 1F, 1G).

To extend our observations from cell culture, we performed intratesticular injection of siRNA for ATG7 or MTOR, and we found that in vivo results were consistent with those in primary cells (Fig. 1H, 1I, 1J). Inhibition or stimulation of autophagy by gene knockdown considerably changed the ABP expression levels in Sertoli cells in the seminiferous tubule (Fig. 1J).

ABP colocalises with LC3 in primary rat Sertoli cells. To investigate how autophagy regulates ABP expression, we first detected ABP gene transcription, and found that either inhibition or stimulation of autophagy did not change the ABP mRNA levels (Fig. 2A). We then used anti-ABP and anti-LC3 antibodies to identify their positions, and the results revealed that $\mathrm{ABP}$ colocalised with $\mathrm{LC} 3$ in primary rat Sertoli cells (Fig. 2B), indicating that ABP is engulfed by the autophagosomes and, therefore, degraded by lysosomes. We also found their colocalisation was promoted by either CQ or rapamycin treatment (Fig. 2B, 2C), suggesting that the ABP expression level is regulated by the autophagic flux.

Clearance of ABP by autophagy is regulated by testosterone. ABP production is promoted by testosterone ${ }^{18,19}$, and testosterone binds to ABP to stimulate its use. In addition, it has been reported that androgen might be involved in autophagy regulation ${ }^{20,21}$. We speculated that testosterone regulates ABP clearance via autophagy in Sertoli cells. Primary Sertoli cells were cultured in complete medium (CM) (medium containing 10\% foetal bovine serum) and treated with or without testosterone; then, the ABP protein and mRNA expression were determined by Western blot (Fig. 3A, 3B), immunocytochemistry (Fig. 3C), and qPCR (Fig. 3D). ABP expression in the supernatants was also tested (Fig. 3E). The results showed that testosterone considerably increased the ABP mRNA and protein levels in primary cells, and testosterone promoted the secretion of $\mathrm{ABP}$ to the supernatant.

To detect whether testosterone regulates autophagy in Sertoli cells, we treated the cells with CM, medium with charcoal stripped serum (MCSS), and MCSS with testosterone. According to the Western blots of LC3B and P62 (P62 is as an adaptor protein in autophagy that interacts with LC3-II; it accumulates when autophagy is inhibited and decreases when autophagy is induced ${ }^{22}$ ), and immunofluorescence (LC3 puncta/cell), we found that removal of testosterone induced autophagy, while treatment with testosterone inhibited autophagy, and there were enhanced inhibitory effects when the concentration of testosterone was increased (Fig. 3F, 3H, Supplementary Fig. S1-S3, S5). We found the changes in P62 expression were more obvious than $\mathrm{LC} 3 \mathrm{~B}$, to determine if $\mathrm{P} 62$ is testosterone responsive, we detected the P62 mRNA expression levels with different doses of testosterone. There were no substantial differences between the groups (Supplementary Fig. S6). We also detected the ABP levels by immunoblots in this experiment and found that testosterone-regulated autophagy affected the ABP expression levels (Fig. 3F, 3G). Besides, results from double immunofluorescence showed that testosterone reduced ABP colocalisation with LC3 under CQ treatment (Fig. 3I, Supplementary Fig. S7).

To provide direct evidence that testosterone regulates ABP degradation, we detected the ABP half-life in response to testosterone and/ or cycloheximide treatment. Cycloheximide is a drug that can inhibit mRNA translation in the cells, and it is widely used in protein degradation analysis ${ }^{23,24}$. The results demonstrated cycloheximide alone didn't affect autophagy, while treatment with testosterone inhibited autophagy (Fig. 3J, Supplementary Fig. S8, S9). Besides, the half-life of ABP was about $6 \mathrm{~h}$ in primary Sertoli cells in the presence of cycloheximide alone, and it was increased to $10 \mathrm{~h}$ with testosterone treatment (Fig. 3J, 3K), suggesting that testosterone considerably decreased the ABP protein degradation.

To investigate how testosterone-regulated autophagy affects ABP expression, primary Sertoli cells were treated with testosterone in the presence of CQ or rapamycin. Interestingly, we found that either the inhibition or promotion of autophagy after testosterone treatment did not change ABP expression in Sertoli cells (Fig. 3L, Supplementary Fig. S11, S12), indicating that testosterone considerably and specifically suppressed the autophagic degradation of ABP.

ABP clearance is independent of stress (hypoxia)-induced autophagy. The above results allow us to speculate that the autophagic clearance of $\mathrm{ABP}$ is selectively regulated by testosterone. Hypoxia and/or nutrition deprivation induce autophagy in most cell types ${ }^{25,26}$. To investigate if stress induces autophagy in primary Sertoli cells, we treated cells with hypoxia or nutrition deprivation. The results showed that only hypoxia induced obvious autophagy in primary Sertoli cells (Fig. 4A), as indicated by the analysis of LC3B and P62 levels. Hypoxia can accelerate the turnover of LC3 protein; to promote the accumulation of LC3, a high concentration of CQ was used before the immunofluorescence assay in both the control and hypoxia groups, and we found that cells treated with hypoxia had more LC3 puncta (Fig. 4B, 4C).

It was recently reported that hypoxia up-regulates $A B P(S H B G)$ in liver cells ${ }^{27}$. We also found that ABP expression in the primary Sertoli cells and their supernatant was considerably increased after exposure to hypoxia (Fig. 4D, 4E, 4F, 4G). To detect if hypoxia-induced autophagy participates in $\mathrm{ABP}$ regulation, we treated cells with hypoxia in the presence of CQ or rapamycin. The results showed that CQ further up-regulated ABP expression in hypoxia (Fig. 4D, 4E, 4G). However, the increase of ABP by CQ under hypoxia was less than that in normal conditions (Fig. 4E), indicating that the autophagic clearance of ABP under hypoxia was lower and that hypoxia-induced autophagy did not clear more ABP. To verify these results, we further treated the cells with testosterone, which inhibited the autophagic degradation of ABP (Fig. 3F). The results demonstrated that after testosterone treatment, neither inhibition nor promotion of hypoxiainduced autophagy changed ABP expression (Fig. 4H, 4I), suggesting that autophagy induced by hypoxia does not clear $\mathrm{ABP}$ and clearance of $\mathrm{ABP}$ is independent of hypoxia-induced autophagy.

\section{Discussion}

In this study, both in vitro and in vivo experiments demonstrate that autophagy regulates ABP expression. Importantly, we found that autophagy reduces $\mathrm{ABP}$ expression through engulfing the protein, and the process seems to be selectively regulated by testosterone. Finally, stress (hypoxia)-induced autophagy does not change the $\mathrm{ABP}$ expression in primary Sertoli cells, which supports the idea that the autophagic degradation of $\mathrm{ABP}$ may be a selectively regulated 
A

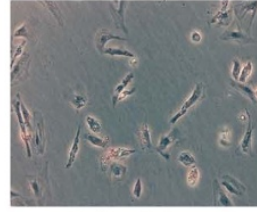

Oil red $\mathrm{O}$

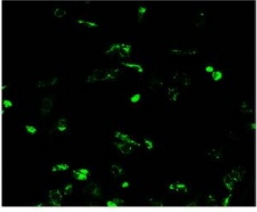

Bodipy $493 / 503$

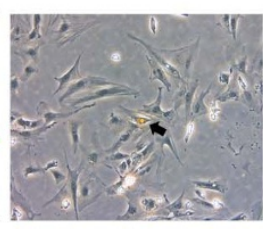

Alkaline phosphatase
B

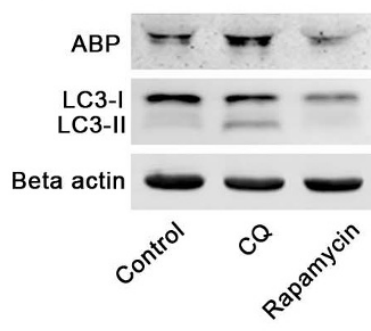

C

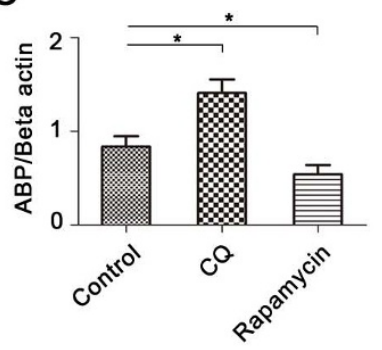

D
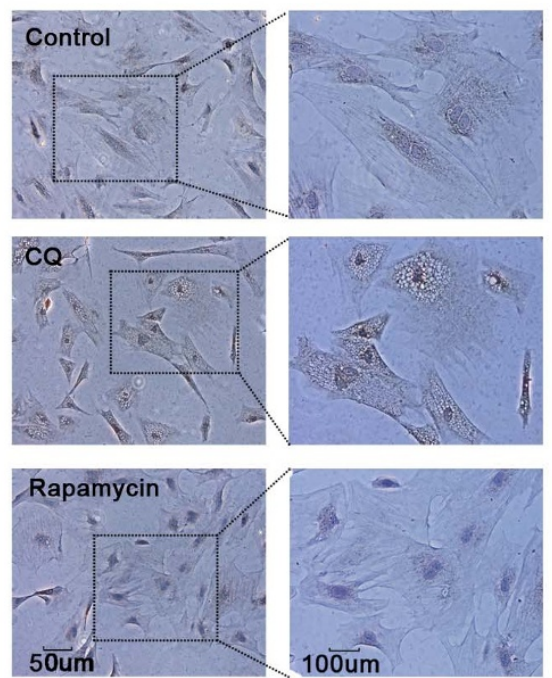

E

G
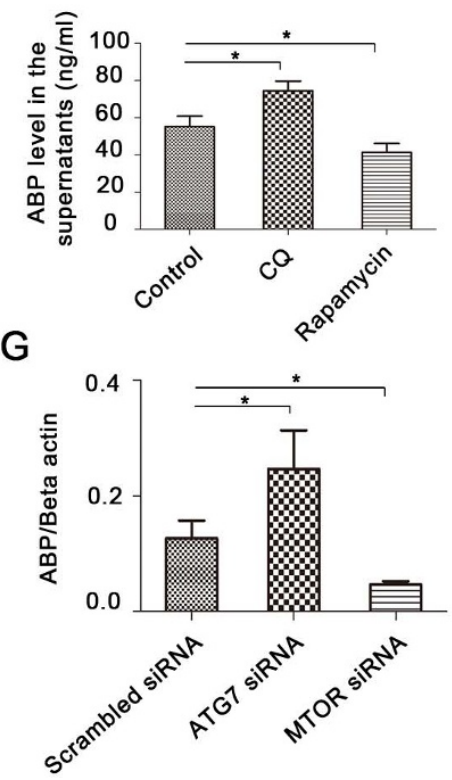

F

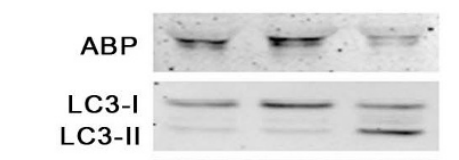

$\mathrm{H}$

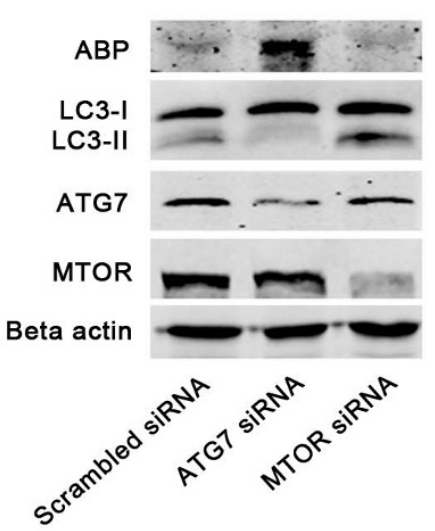

I

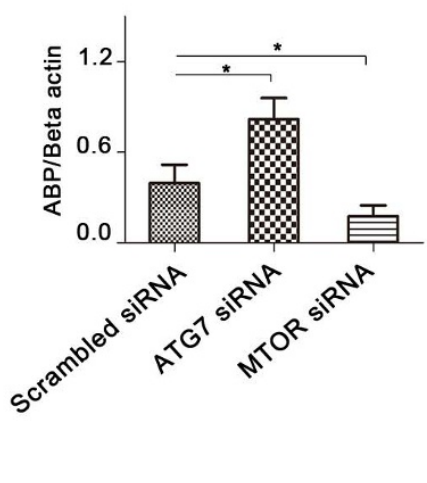

J

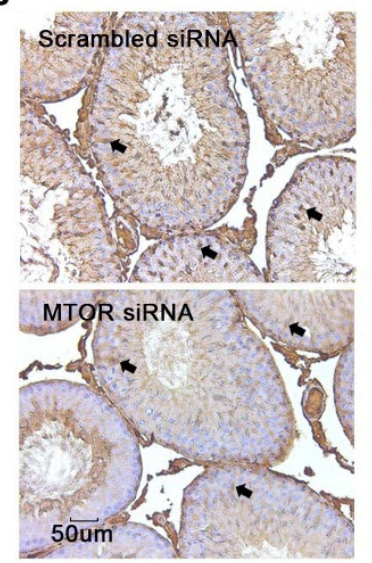

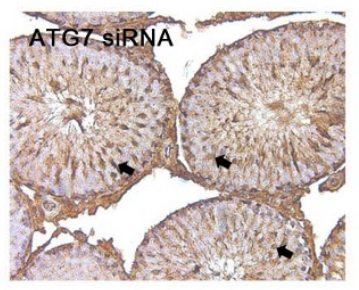

Figure 1 Autophagy regulates ABP expression in the primary Sertoli cells and rat testes. (A), Cells isolated from 20-day-old rat testis were stained with oil red O, bodipy 493/503 or alkaline phosphatase detection kit, and pictures were taken with a fluorescence microscope. Black arrow: germ cell. (B-E), Rat primary Sertoli cells were treated with CQ $(50 \mathrm{uM})$ or rapamycin $(10 \mathrm{nM})$ for $24 \mathrm{~h}$, and ABP expression in the cells was assessed with Western blot (B) and immunocytochemistry (D). ABP in the supernatants was determined by ELISA (E) $(n=3, * p<0.05)$. Densitometric analysis of the bands in ABP is shown in (C) (mean $\pm \mathrm{SD}$ of independent experiments, $\left.\mathrm{n}=3,{ }^{*} \mathrm{p}<0.05\right)$. LC3B was assessed by Western blot (B). (F-G), Cells were treated with scrambled siRNA, ATG7 siRNA, or MTOR siRNA for $48 \mathrm{~h}$, and ABP, LC3B, ATG7, MTOR and Beta actin were determined by Western blots (F), and densitometric analysis of ABP immunoblots is shown $(\mathrm{G})\left(\mathrm{n}=3,{ }^{*} \mathrm{p}<0.05\right)$. (H-J), The rat testis was directly injected with 50 ul mixed liquid of in vivojetPEI and methylation modified siRNA ( $5 \mathrm{nmol})$ as indicated; primary Sertoli cells were isolated after 2 days, and ABP was determined by Western blot $(\mathrm{H})$ and immunohistochemistry $(\mathrm{J})$ using adjacent tissue. Densitometric analysis of ABP immunoblots is shown $(\mathrm{I})\left(\mathrm{n}=3,{ }^{*} \mathrm{p}<0.05\right)$. Haematoxylin was used to stain the nuclei (blue). Black arrows: Sertoli cells.

process. To the best of our knowledge, we are the first to reveal that autophagy clears ABP in Sertoli cells; testosterone increases ABP expression not only by promoting its synthesis, and suppressing its autophagic degradation.
Sertoli cells are crucial support cells in the seminiferous tubule. To maintain a stable spermatogenic microenvironment and to support the growth of spermatogenic cells, Sertoli cells secrete many important proteins, such as anti-Müllerian hormone, inhibin, oestradiol 


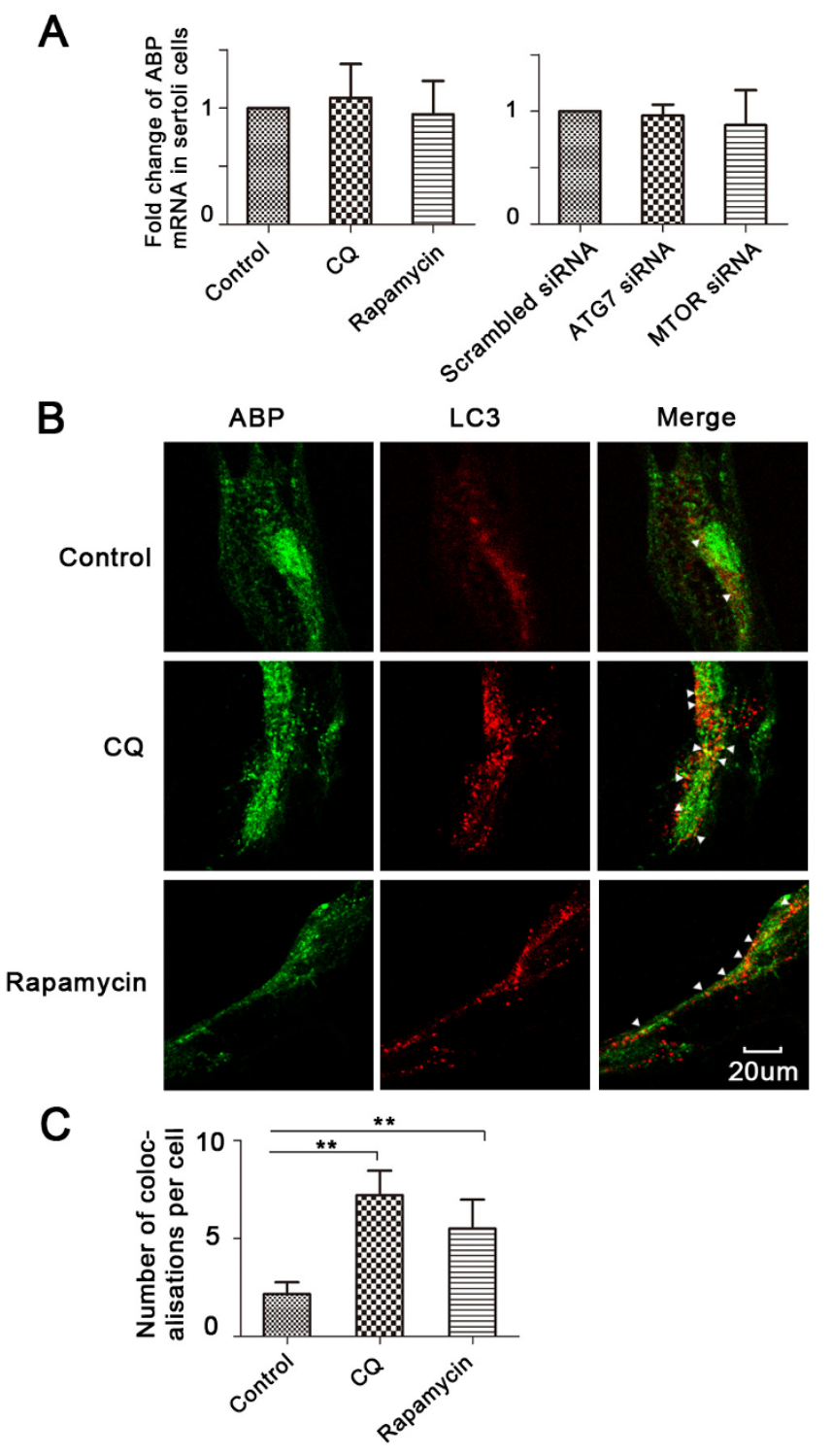

Figure $2 \mid$ ABP colocalises with LC3 in primary Sertoli cells. (A), Rat primary Sertoli cells were treated with CQ $(50 \mathrm{uM})$ or rapamycin $(10 \mathrm{nM})$ for $24 \mathrm{~h}(\mathrm{n}=3)$, or treated with ATG7 or MTOR siRNA for $48 \mathrm{~h}(\mathrm{n}=3)$, ABP mRNA was determined by qPCR. (B-C), Cells were treated with CQ or rapamycin for $24 \mathrm{~h}$, and ABP and LC3 were assessed with double immunofluorescence (B). The average number of colocalisations per cell was calculated from 10 random fields (approximately 200 cells) $(C)(n=3$, $* * \mathrm{p}<0.01)$.

and $\mathrm{ABP}$. $\mathrm{ABP}$ can increase the concentration of testosterone in the seminiferous tubule to promote spermatogenesis. However, it has not previously been shown how ABP expression is regulated. Autophagy is an adaptive cellular response that is designed to protect the cell through eliminating cellular components by lysosomal degradation ${ }^{28}$. LC3 is the most widely monitored autophagy-related protein $^{29}$. When autophagy is induced, there may be increases in the LC3-II/LC3-I ratios, decreases in both LC3-I and LC3-II, or a decrease in LC3-II relative to LC3-I if LC3-II degradation via lysosomal turnover is rapid. $\mathrm{P} 62$ is an adaptor protein that is required for the formation of ubiquitinated protein aggregates ${ }^{30}$. The interaction of P62 with both LC3 and ubiquitinated proteins can mediate the delivery of those aggregates to the autophagy system ${ }^{31}$. As a result, P62 accumulates when autophagy is inhibited, and it decreases when autophagy is induced $^{22}$. The use of CQ to block autophagy in cultured cells is technically easy and has been widely accepted. CQ can inac- tivate lysosomal hydrolases by inhibiting lysosomal acidification, thereby increasing the LC3-II expression level and LC3-II/LC3-I ratios $^{32}$. The MTOR inhibitor, rapamycin, can induce autophagy and promote the conversion of LC3-I to LC3-II, decreasing LC3-I expression and increasing the LC3-II/LC3-I ratios. Recent studies revealed that autophagy plays a central role in protein clearance and secretion ${ }^{33,34}$. In this context, we speculated that autophagy clears ABP in Sertoli cells, and, indeed we found that autophagy degrades ABP.

The conventional view about testosterone and $\mathrm{ABP}$ is that testosterone increases ABP synthesis and secretion ${ }^{18,19,35}$. However, until our study, it was unknown if testosterone regulates ABP clearance. Testosterone can bind to ABP to promote its use and reduce ABP accumulation in Sertoli cells. Also, it has been reported that androgen regulates autophagy through androgen receptor-mediated up-regulation of $78 / \mathrm{BiP}^{20}$; therefore, we speculated that testosterone may reduce the degradation of excess ABP through androgen receptor-mediated autophagy. Our results show that testosterone inhibits autophagy and up-regulates ABP expression. In addition, CQ or rapamycin can promote or inhibit $\mathrm{ABP}$ expression. However, after treatment with testosterone, ABP expression is not affected by CQ or rapamycin, indicating that testosterone might be a specific switch controller of the autophagic clearance of ABP. There might be a threshold, and when the testosterone concentration is below the threshold, the switch is turned on. Clearance of ABP by autophagy can be enhanced or inhibited by rapamycin or CQ. In contrast, adding testosterone up to the threshold turns off the switch, and the clearance of $\mathrm{ABP}$ is no longer affected by the drug that regulates autophagy. Therefore, it seems that the autophagic clearance of $\mathrm{ABP}$ is selectively regulated by testosterone.

Our results demonstrate the clearance of ABP is independent of stress (hypoxia)-induced autophagy, which further supports the idea that the autophagic degradation of ABP is a selectively controlled process. Autophagy can be stimulated by some stress factors, such as hypoxia or starvation ${ }^{36,37}$. Stress-induced autophagy degrades self proteins, maintains energy homeostasis and combats harmful factors $^{36}$. However, in this study, we found that hypoxia-induced autophagy does not clear ABP. On the contrary, hypoxia up-regulates ABP expression. A possible explanation is that hypoxia increases testosterone through a VEGF pathway ${ }^{38,39}$, and testosterone stimulates $\mathrm{ABP}$ expression. Therefore, hypoxia might up-regulate $\mathrm{ABP}$ in an indirect way. It is possible that the induction of $\mathrm{ABP}$ by hypoxia is an adaptive response to hypoxia-induced cell death, maintaining a high concentration of ABP in the seminiferous tubule.

Autophagy is involved in spermatogenesis, and previous studies focused on the relationship between autophagy and germ cell death, sperm function, or testosterone secretion ${ }^{40-42}$. However, there is a paucity of data discussing autophagy and ABP in spermatogenesis. It has been reported that CQ has some toxic effects on reproductive function ${ }^{43}$, but the detailed mechanisms are still not clear. According to the results of this study, a possible reason is the dysregulation of $\mathrm{ABP}$ production induced by $\mathrm{CQ}$.

In summary, we determined that in rat Sertoli cells, ABP can be cleared by autophagy. Of note, autophagy degrades ABP by engulfing the protein, and the process seems to be selectively regulated by testosterone, which can prolong ABP biological half-life by inhibiting autophagy. Finally, we found that ABP clearance is independent of stress (hypoxia)-induced autophagy. Although further studies are required to understand the role of autophagic clearance of ABP in Sertoli cells, these results have critical implications about the mechanisms by which testosterone regulates $\mathrm{ABP}$ expression as well as that by which ABP clearance is regulated.

\section{Methods}

Ethics statement. All procedures have been conducted according to the Declaration of Helsinki and $\mathrm{NIH}$ guidelines. All animal experiments were performed in 
A

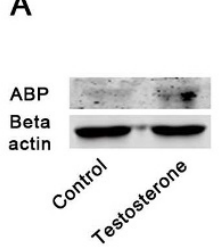

B

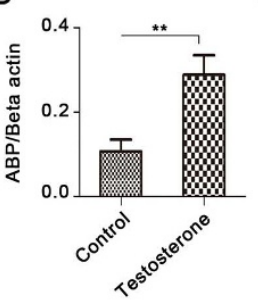

C

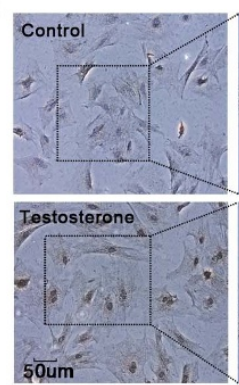

F

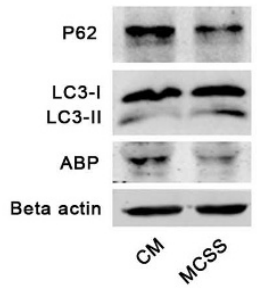

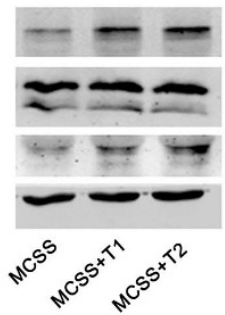

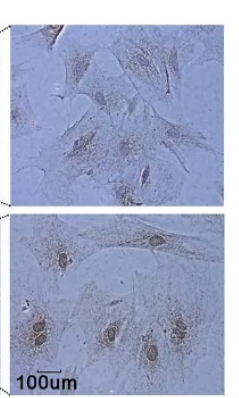

D

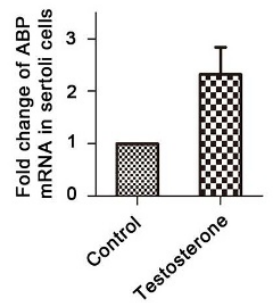

E

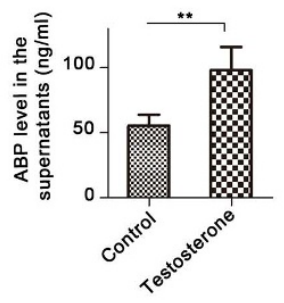

H

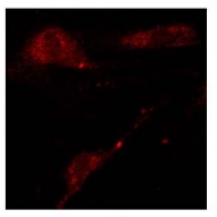

CM

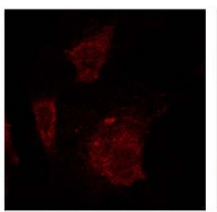

McSS+T1

J

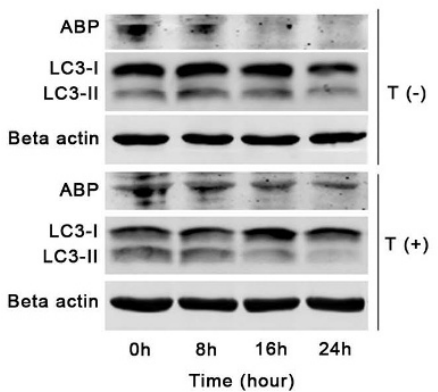

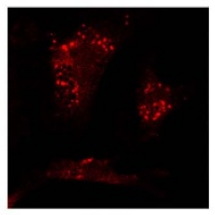

MCSS

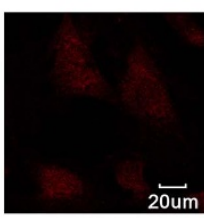

MCSS+T2
I

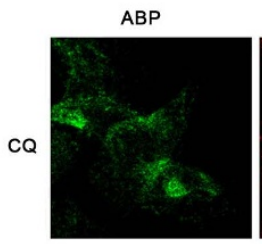

G

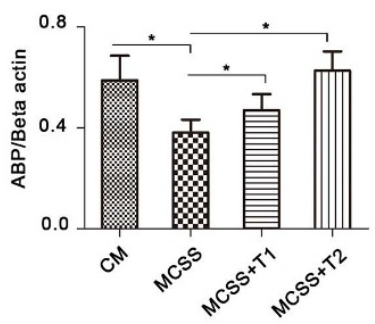

LC3
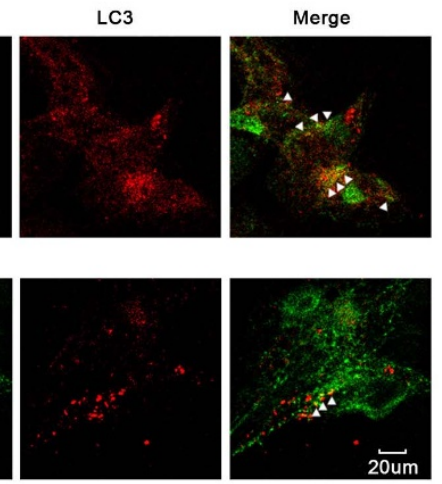

L

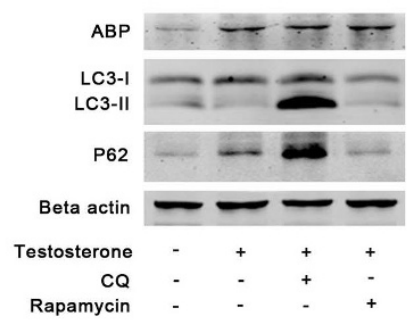

Figure 3 Testosterone up-regulates ABP expression through promoting its synthesis and suppressing autophagic degradation. (A-E), Rat primary Sertoli cells were cultured in CM and treated with testosterone for $24 \mathrm{~h}$, and ABP expression in the cells was determined with Western blot (A), immunocytochemistry $(\mathrm{C})$ and $\mathrm{qPCR}(\mathrm{D})(\mathrm{n}=3)$. The ABP expression level in the supernatant was assessed by ELISA (E) ( $\mathrm{n}=3, * * \mathrm{p}<0.01)$. The densitometric analysis of ABP immunoblots is shown in (B) $\left(\mathrm{n}=3,{ }^{* *} \mathrm{p}<0.01\right)$. (F-H), Cells were cultured in CM, MCSS, MCSS+T1 and MCSS + T2 for $24 \mathrm{~h}$, and LC3B, P62 and ABP were determined by Western blots (F). LC3B was also assessed by immunofluorescence (H). Densitometric analysis of the bands in ABP immunoblots is shown in $(\mathrm{G})\left(\mathrm{n}=3,{ }^{*} \mathrm{p}<0.05\right)$. The blots of LC3B and Beta actin in Fig. 3F were cropped and full-length blots are presented in Supplementary Figure S4. The average number of LC3 puncta per cell was calculated in Supplementary Figure S5. (I), Cells were treated with $\mathrm{CQ}(50 \mathrm{uM})$ in the presence or absence of testosterone $(10 \mathrm{nM})$, and the ABP and LC3 expression levels were assessed with double immunofluorescence after $24 \mathrm{~h}$. The average number of ABP-LC3 colocalizations per cell was calculated in Supplementary Figure S7. (J-K), Cells were treated with cycloheximide $(150 \mathrm{uM})$ in the presence or absence of testosterone $(10 \mathrm{nM})$ as indicated (T: Testosterone) for different time periods, the ABP and LC3 expression were determined by Western blots $(\mathrm{J})$. The relative ABP levels were determined by measuring the density of protein band, and normalized to Beta actin $(\mathrm{K})\left(\mathrm{n}=3,{ }^{*} \mathrm{p}<0.05\right)$. The relative ABP protein level at time zero was termed as 1 . The blots of ABP and Beta actin of the testosterone $(+)$ group were cropped and full-length blots are presented in Supplementary Figure S10. (L), Cells were cultured in CM and treated with testosterone $(10 \mathrm{nM}), \mathrm{CQ}(50 \mathrm{uM})$ or rapamycin $(10 \mathrm{nM})$, as indicated. ABP, LC3B, P62 and Beta actin were determined by Western blots after 24 h. CM: Complete medium (medium with 10\% foetal bovine serum); MCSS: Medium with 10\% charcoal stripped serum. T1: Testosterone (10 nM); and T2: Testosterone $(50 \mathrm{nM})$. 
A
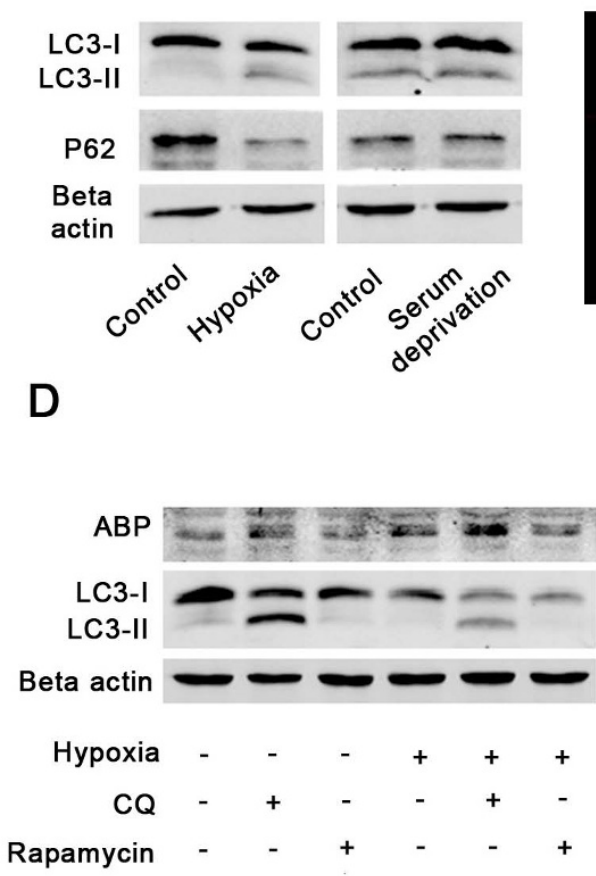

B

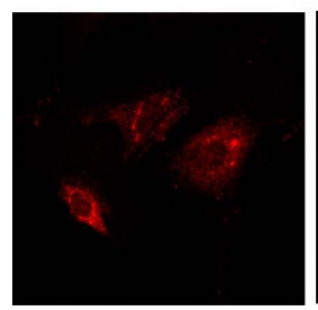

Control

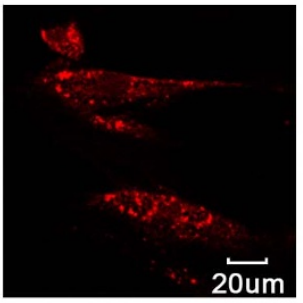

Hypoxia

C

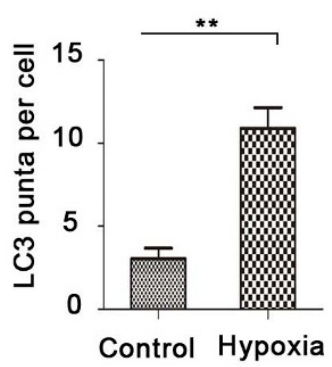

F

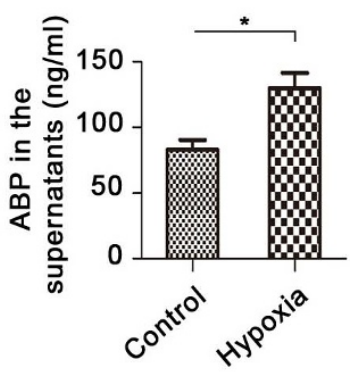

G
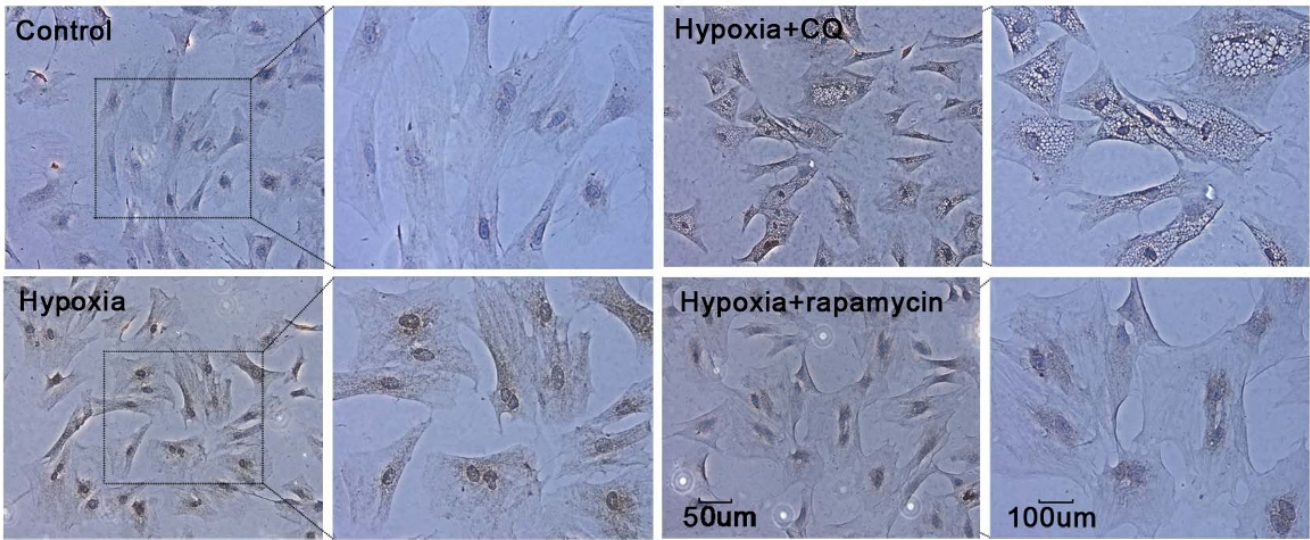

$\mathrm{H}$

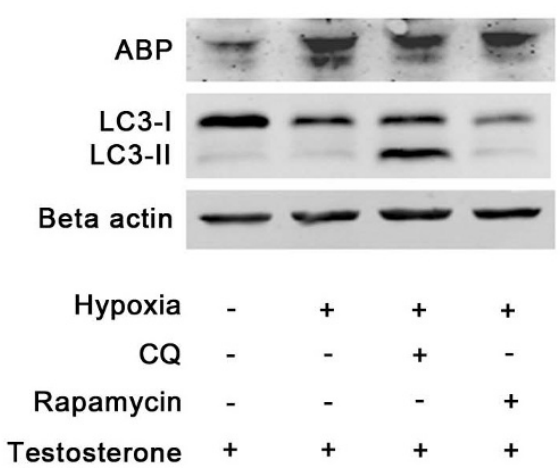

I
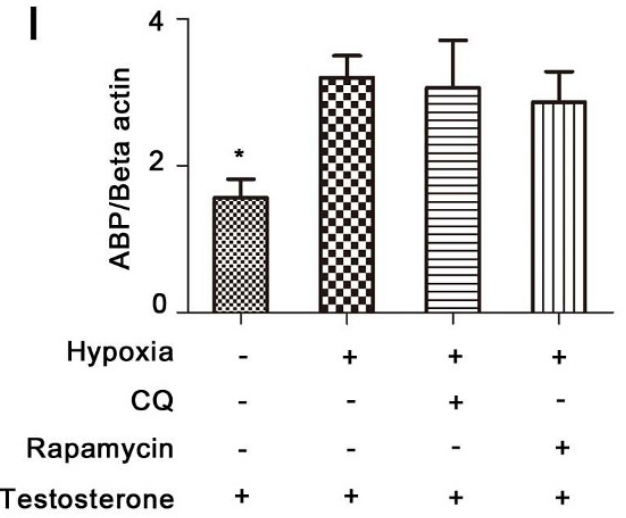

Figure $4 \mid$ Stress-induced autophagy does not degrade ABP. (A-C), Primary Sertoli cells were cultured in hypoxia (1\%) or serum deprivation medium for $24 \mathrm{~h}, \mathrm{LC} 3 \mathrm{~B}$ and P62 were determined by Western blots (A). LC3B was also assessed by immunofluorescence (B). A high concentration of CQ (100 uM) was used before the immunofluorescence assay to promote the accumulation of LC3. The average number of puncta per cell was calculated from 10 random fields (C) $\left(\mathrm{n}=3,{ }^{* *} \mathrm{p}<0.01\right)$. (D-G), Cells were treated with CQ $(50 \mathrm{uM})$ or rapamycin $(10 \mathrm{nM})$ in the presence or absence of hypoxia for $24 \mathrm{~h}$. ABP, LC3B and Beta actin were determined by Western blots (D). The bands in ABP immunoblots were analysed (E) $\left(\mathrm{n}=3,{ }^{*} \mathrm{p}<0.05, * * \mathrm{p}<0.01\right)$. ELISA was used to determine the ABP expression in the supernatants $(\mathrm{F})\left(\mathrm{n}=3,{ }^{*} \mathrm{p}<0.05\right)$. ABP was also assessed by immunocytochemistry $(\mathrm{G})$. (H-I), Cells were exposed to hypoxia and treated with testosterone $(10 \mathrm{nM})$, CQ $(50 \mathrm{uM})$ or rapamycin $(10 \mathrm{nM})$ as indicated, ABP and LC3B were determined by immunoblots $(\mathrm{H})$. The densitometric analysis of the bands in ABP immunoblots is shown in (I) ( $\mathrm{n}=3$, ${ }^{*} \mathrm{p}<0.05$ when compared with any other group). 
accordance with the NIH Guide for the Care and Use of Laboratory Animals. The study was also approved by the institutional review board of Renji Hospital.

Isolation and culturing of sertoli cells. Male Sprague-Dawley rats were purchased from Shanghai SLAC laboratory Animal Co., Ltd. A total of 20 Sprague-Dawley rats (18-22 days) were used for primary Sertoli cell isolation. The isolation method was as previously described with minor modification $s^{17}$. Briefly, after the tunica albuginea was removed, all testes were placed into F12/DMEM $(1: 1)(11039$, GIBCO) and washed 2 times. The testes were cut into $\sim 1$-mm pieces and washed to remove contaminating blood cells, they were then centrifuged at $800 \mathrm{~g}$ for $2 \mathrm{~min}$. Testes were resuspended in F12/DMEM with 0.1\% trypsin (25300, GIBCO) and then placed in a shaking water bath at $60 \mathrm{osc} / \mathrm{min}$ for $30 \mathrm{~min}$, to release Leydig cells. The bottom seminiferous tubules were transferred to a vial with F12/DMEM supplemented with $10 \%$ foetal bovine serum (10099, Gibco) and washed until F12/DMEM was clear. The supernatant was discarded and, cells were resuspended in F12/DMEM containing $0.1 \%$ collagenase V (C9263, Sigma-Aldrich) and placed in a shaking water bath at $60 \mathrm{osc} / \mathrm{min}$ for $40 \mathrm{~min}$, to remove peritubular myoid cells. Cells were washed by centrifugation at $800 \mathrm{~g}$ two times, resuspended cells with F12/DMEM containing $0.1 \%$ hyaluronidase ( $\mathrm{H} 3506$, Sigma-Aldrich) and placed in the shaking water bath for $30 \mathrm{~min}$, to break down hyaluronic acid. Cells were washed twice by centrifugation at $800 \mathrm{~g}$, resuspended and incubated with F12/DMEM supplemented with $50 \mathrm{U} / \mathrm{ml}$ penicillin, $50 \mu \mathrm{g} / \mathrm{ml}$ streptomycin (10378, GIBCO), and 10\% charcoal stripped foetal bovine serum (12676, GIBCO) or 10\% foetal bovine serum. Forty-eight hours after plating, cells were treated with $20 \mathrm{mM}$ Tris ( $\mathrm{pH} 7.4$ ) for $2.5 \mathrm{~min}$ to remove residual germ cells. After two washes, cells were incubated in a humidified $\mathrm{CO} 2$ incubator at $35^{\circ} \mathrm{C}$ with $95 \%$ air and $5 \% \mathrm{CO}$. If cells were cultured in hypoxia, the oxygen was maintained at $1 \%$.

Chemical Reagents, siRNA, in vitro and in vivo Transfection. Chloroquine (CQ), oil red $\mathrm{O}$, testosterone and rapamycin were obtained from Sigma-Aldrich, MO, USA (C6628, O0625, T6147, 37094). Cycloheximide was purchased from Chengdu Ai Keda Chemical Technology Company (66-81-9). Methylation modified siRNA against rat ATG7 (5'-GGAUACAAGCUUGGCUGCUACUUCU-3 ${ }^{\prime}$ ) and MTOR (5'-CGGCAGACUGGCUCUUGCUCAUAAA-3') were purchased from RiboBio, Guangzhou, China. Transfection of nucleic acids was performed using Lipofectamine 2000 (11668, Life Technologies) and in vivo-jetPEI (201-10G, Polyplus) according to the manufacturer's instructions. For intra-testicular injection, the rats (8 weeks) were randomly divided into 3 groups (Scrambled siRNA group, $\mathrm{n}=3$; ATG7 siRNA group, $\mathrm{n}=3$; and MTOR siRNA group, $\mathrm{n}=3$ ). A mixed liquid (50 ul) with $5 \mathrm{nmol}$ siRNA and in vivo-jetPEI was directly injected into the rat testis according to the manufacturer's instructions. Rats were sacrificed after 2 days, and ABP was determined by immunohistochemistry in testis tissue. Primary Sertoli cells from the adjacent testis tissue were also isolated, and ABP, ATG7, MTOR, and LC3B were detected with Western blots analysis.

RNA Isolation, cDNA Synthesis and Quantitative Real-time PCR (qPCR). Total RNA isolation, cDNA synthesis and qPCR were performed as previously described $^{44,45}$. The primers for qPCR are listed as follows: rat GAPDH $\left(5^{\prime}-3^{\prime}\right)$ F: GGCACAGTCAAGGCTGAGAATG, R: ATGGTGGTGAAGACGCCAGTA; rat SHBG (ABP) (5'-3') F: ATGTGGACCTGCAACCTGGAC, R: TGCTCCATCCACCAGCTTAAATC and; rat SQSTM1 (P62) (5'-3') F CCTCAGCCCTCTAGGCATCG, R: GTACAGGGCAGCTTCCTTCA.

Protein extraction and western blot. Protein extraction and Western blot were performed as previously described ${ }^{26}$. Protein from primary Sertoli cells was isolated with M-PER Mammalian Protein Extraction Reagent (78501, Thermo Scientific). Rabbit anti-LC3B (L7543), ACTB (beta actin) (8457), P62/SQSTM1 (5114), ATG7 (8558) and MTOR (2983) were purchased from Sigma-Aldrich Co. LLC. Anti-SHBG (ABP) (SC-32891) was obtained from Santa Cruz Biotechnology, Inc.

Oil red O, Bodipy 493/503 and Alkaline phosphatase staining. For oil red $O$ and bodipy $493 / 503$ staining, cells were washed with PBS, fixed with $4 \%$ formaldehyde for $20 \mathrm{~min}$, and stained with oil red O (0.2\%) or bodipy 493/503 (D3922, Life Technologies. stock concentration $2 \mathrm{mg} / \mathrm{ml}$, working solution $1: 200$ dilution) for $15 \mathrm{~min}$ at room temperature. The alkaline phosphatase kit was purchased from Shanghai Fushen Biotechnology Company and, staining was performed according to the manufacturer's instructions.

Immunofluorescence and confocal microscopy. Cells were washed with PBS and fixed in ice-cold ethanol for $15 \mathrm{~min}$ at $-20^{\circ} \mathrm{C}$. After blocking for $20 \mathrm{~min}$, cells were incubated in the primary antibodies at $4^{\circ} \mathrm{C}$ overnight, and Alexa Fluor 594 goat antimouse IgG (A11012, Invitrogen) and Alexa Fluor 488 goat anti-rabbit IgG (A11008, Invitrogen) served as the secondary antibodies. To prepare the glass histological slide, rat testis was fixed in Bouin's fixative, embedded in paraffin, and sectioned at $3 \mu \mathrm{m}$ thickness. Anti-SHBG (ABP) (sc-32891) and Anti-LC3B (sc-376404) were purchased from Santa Cruz Biotechnology, Inc. Immunofluorescence was detected with confocal microscopy as previously described ${ }^{26}$.

Immunocytochemistry, Immunohistochemistry and Enzyme-Linked Immunosorbent Assays (ELISA). Immunocytochemistry and immunohistochemistry was performed as previously described ${ }^{46}$. Briefly, cells or tissue were incubated in the primary antibodies at $4{ }^{\circ} \mathrm{C}$ overnight and the secondary antibodies for $30 \mathrm{~min}$ at $37^{\circ} \mathrm{C}$; then, they were visualized with diaminobenzadine (DAB) and counterstained with hematoxylin. The ABP levels in the supernatants were assessed by an ELISA kit for rats (CSB-E12118r, Cusabio) according to the manufacturer's instructions.

Statistical analysis. Data analyses were conducted with SPSS 16.0 (SPSS, IL, USA). The bar charts were plotted with GraphPad-Prism5 (GraphPad, CA, USA). Data are presented as the mean \pm standard deviation (SD) and analysed by Student's t test. All tests were 2 -tailed, and $\mathrm{p}<0.05$ was considered statistically significant. Each experiment was performed in triplicate.

1. Della-Maria, J., Gerard, A., Franck, P. \& Gerard, H. Effects of androgen-binding protein $(\mathrm{ABP})$ on spermatid Tnpl gene expression in vitro. Mol Cell Endocrinol 198, 131-141 (2002).

2. Selva, D. M. et al. Meiotic arrest and germ cell apoptosis in androgen-binding protein transgenic mice. Endocrinology 141, 1168-1177, doi:10.1210/ endo.141.3.7383 (2000)

3. Janne, M., Deol, H. K., Power, S. G., Yee, S. P. \& Hammond, G. L. Human sex hormone-binding globulin gene expression in transgenic mice. Mol Endocrinol 12, 123-136, doi:10.1210/mend.12.1.0050 (1998).

4. Selva, D. M. et al. Human sperm sex hormone-binding globulin isoform: characterization and measurement by time-resolved fluorescence immunoassay. J Clin Endocrinol Metab 90, 6275-6282, doi:10.1210/jc.2005-1192 (2005).

5. Selva, D. M. \& Hammond, G. L. Human sex hormone-binding globulin is expressed in testicular germ cells and not in sertoli cells. Horm Metab Res 38, 230-235, doi:10.1055/s-2006-925336 (2006)

6. Hermo, L., Barin, K. \& Oko, R. Androgen binding protein secretion and endocytosis by principal cells in the adult rat epididymis and during postnatal development. J Androl 19, 527-541 (1998).

7. Tindall, D. J., Mena, C. R. \& Means, A. R. Hormonal regulation of androgenbinding protein in hypophysectomized rats. Endocrinology 103, 589-594, doi:10.1210/endo-103-2-589 (1978).

8. Karl, A. F. \& Griswold, M. D. Actions of insulin and vitamin A on Sertoli cells. Biochem J 186, 1001-1003 (1980).

9. Aleem, M. et al. Estradiol affects androgen-binding protein expression and fertilizing ability of spermatozoa in adult male rats. Mol Cell Endocrinol 253, 1-13, doi:10.1016/j.mce.2006.01.014 (2006).

10. Li, H. et al. Effect of surgically induced varicocele on testicular blood flow and Sertoli cell function. Urology 53, 1258-1262 (1999).

11. Dimitriadis, F. et al. Effects of phosphodiesterase-5 inhibitor vardenafil on testicular androgen-binding protein secretion, the maintenance of foci of advanced spermatogenesis and the sperm fertilising capacity in azoospermic men. Andrologia 44 Suppl 1, 144-153, doi:10.1111/j.1439-0272.2010.01153.x (2012).

12. Tang, X. M., Zhang, H. X. \& Yi, J. [Leydig cells-a normal cell model of cellular autophagy]. Shi Yan Sheng Wu Xue Bao 25, 39-47 (1992).

13. Yefimova, M. G. et al. A chimerical phagocytosis model reveals the recruitment by Sertoli cells of autophagy for the degradation of ingested illegitimate substrates. Autophagy 9, 653-666, doi:10.4161/auto.23839 (2013).

14. Chen, Y., Zhou, Y., Wang, X., Qian, W. \& Han, X. Microcystin-LR induces autophagy and apoptosis in rat Sertoli cells in vitro. Toxicon 76, 84-93, doi:10.1016/j.toxicon.2013.09.005 (2013).

15. Eid, N., Ito, Y. \& Otsuki, Y. Enhanced mitophagy in Sertoli cells of ethanol-treated rats: morphological evidence and clinical relevance. J Mol Histol 43, 71-80, doi:10.1007/s10735-011-9372-0 (2012).

16. Corcelle, E. et al. Disruption of autophagy at the maturation step by the carcinogen lindane is associated with the sustained mitogen-activated protein kinase/ extracellular signal-regulated kinase activity. Cancer Res 66, 6861-6870, doi:10.1158/0008-5472.CAN-05-3557 (2006).

17. Mruk, D. D. \& Cheng, C. Y. An in vitro system to study Sertoli cell blood-testis barrier dynamics. Methods Mol Biol 763, 237-252, doi:10.1007/978-1-61779-1918_16 (2011).

18. Danzo, B. J., Pavlou, S. N. \& Anthony, H. L. Hormonal regulation of androgenbinding protein in the rat. Endocrinology 127, 2829-2838, doi:10.1210/endo-1276-2829 (1990).

19. Hadley, M. A., Djakiew, D., Byers, S. W. \& Dym, M. Polarized secretion of androgen-binding protein and transferrin by Sertoli cells grown in a bicameral culture system. Endocrinology 120, 1097-1103, doi:10.1210/endo-120-3-1097 (1987).

20. Bennett, H. L., Fleming, J. T., O’Prey, J., Ryan, K. M. \& Leung, H. Y. Androgens modulate autophagy and cell death via regulation of the endoplasmic reticulum chaperone glucose-regulated protein $78 / \mathrm{BiP}$ in prostate cancer cells. Cell Death Dis 1, e72, doi:10.1038/cddis.2010.50 (2010).

21. Li, M. et al. Autophagy protects $\mathrm{LNCaP}$ cells under androgen deprivation conditions. Autophagy 4, 54-60 (2008).

22. Bjorkoy, G. et al. Monitoring autophagic degradation of p62/SQSTM1. Methods Enzymol 452, 181-197, doi:10.1016/S0076-6879(08)03612-4 (2009).

23. Eskandani, M., Abdolalizadeh, J., Hamishehkar, H., Nazemiyeh, H. \& Barar, J. Galbanic acid inhibits HIF-1alpha expression via EGFR/HIF-1alpha pathway in cancer cells. Fitoterapia, doi:10.1016/j.fitote.2014.12.003 (2014). 
24. Liu, L. Z. et al. Acacetin inhibits VEGF expression, tumor angiogenesis and growth through AKT/HIF-1alpha pathway. Biochem Biophys Res Commun 413, 299-305, doi:10.1016/j.bbrc.2011.08.091 (2011).

25. Steiger-Barraissoul, S. \& Rami, A. Serum deprivation induced autophagy and predominantly an AIF-dependent apoptosis in hippocampal HT22 neurons. Apoptosis 14, 1274-1288, doi:10.1007/s10495-009-0396-9 (2009).

26. Ma, Y. et al. Biphasic regulation of autophagy by miR-96 in prostate cancer cells under hypoxia. Oncotarget 5, 9169-9182 (2014).

27. Lu, X., Yu, R. M., Murphy, M. B., Lau, K. \& Wu, R. S. Hypoxia disrupts gene modulation along the brain-pituitary-gonad (BPG)-liver axis. Ecotoxicol Environ Saf 102, 70-78, doi:10.1016/j.ecoenv.2014.01.017 (2014).

28. Kroemer, G., Marino, G. \& Levine, B. Autophagy and the integrated stress response. Mol Cell 40, 280-293, doi:10.1016/j.molcel.2010.09.023 (2010).

29. Klionsky, D. J. et al. Guidelines for the use and interpretation of assays for monitoring autophagy. Autophagy 8, 445-544 (2012).

30. Pankiv, S. et al. p62/SQSTM1 binds directly to Atg8/LC3 to facilitate degradation of ubiquitinated protein aggregates by autophagy. J Biol Chem 282, 24131-24145, doi:10.1074/jbc.M702824200 (2007).

31. Bjorkoy, G., Lamark, T. \& Johansen, T. p62/SQSTM1: a missing link between protein aggregates and the autophagy machinery. Autophagy 2, 138-139 (2006).

32. Palmeira dos Santos, C. et al. Comparative study of autophagy inhibition by $3 \mathrm{MA}$ and CQ on Cytarabineinduced death of leukaemia cells. J Cancer Res Clin Oncol 140, 909-920, doi:10.1007/s00432-014-1640-4 (2014).

33. Chatterjee, C. \& Sparks, D. L. Hepatic lipase release is inhibited by a purinergic induction of autophagy. Cell Physiol Biochem 33, 883-894, doi:10.1159/ 000358661 (2014)

34. Chatterjee, C. \& Sparks, D. L. Extracellular nucleotides inhibit insulin receptor signaling, stimulate autophagy and control lipoprotein secretion. PLoS One 7, e36916, doi:10.1371/journal.pone.0036916 (2012).

35. Ritzen, E. M., Hagenas, L., Hansson, V. \& Frensh, F. S. In vitro synthesis of testicular androgen binding protein (ABP): stimulation by FSH and androgen. Curr Top Mol Endocrinol 2, 353-366 (1975).

36. Kuma, A. et al. The role of autophagy during the early neonatal starvation period. Nature 432, 1032-1036, doi:10.1038/nature03029 (2004).

37. Filomeni, G., De Zio, D. \& Cecconi, F. Oxidative stress and autophagy: the clash between damage and metabolic needs. Cell Death Differ, doi:10.1038/ cdd.2014.150 (2014).

38. Hwang, G. S., Wang, S. W., Tseng, W. M., Yu, C. H. \& Wang, P. S. Effect of hypoxia on the release of vascular endothelial growth factor and testosterone in mouse TM3 Leydig cells. Am J Physiol Endocrinol Metab 292, E1763-1769, doi:10.1152/ ajpendo.00611.2006 (2007).

39. Madrid, E. et al. Effect of normobaric hypoxia on the testis in a murine model Andrologia 45, 332-338, doi:10.1111/and.12019 (2013).

40. Bustamante-Marin, X., Quiroga, C., Lavandero, S., Reyes, J. G. \& Moreno, R. D. Apoptosis, necrosis and autophagy are influenced by metabolic energy sources in cultured rat spermatocytes. Apoptosis 17, 539-550, doi:10.1007/s10495-0120709-2 (2012).
41. Wang, H. et al. Atg7 is required for acrosome biogenesis during spermatogenesis in mice. Cell Res 24, 852-869, doi:10.1038/cr.2014.70 (2014).

42. Li, W. R. et al. Autophagic deficiency is related to steroidogenic decline in aged rat Leydig cells. Asian J Androl 13, 881-888, doi:10.1038/aja.2011.85 (2011).

43. Roy, L. D., Mazumdar, M. \& Giri, S. Effects of low dose radiation and vitamin C treatment on chloroquine-induced genotoxicity in mice. Environ Mol Mutagen 49, 488-495, doi:10.1002/em.20408 (2008).

44. Ma, Y., Dai, H., Kong, X. \& Wang, L. Impact of thawing on reference gene expression stability in renal cell carcinoma samples. Diagn Mol Pathol 21, 157-163, doi:10.1097/PDM.0b013e31824d3435 (2012).

45. Ma, Y. et al. Renal tissue thawed for 30 minutes is still suitable for gene expression analysis. PLoS One 9, e93175, doi:10.1371/journal.pone.0093175 (2014).

46. Forges, T., Gerard, A., Hess, K., Monnier-Barbarino, P. \& Gerard, H. Expression of sex hormone-binding globulin (SHBG) in human granulosa-lutein cells. Mol Cell Endocrinol 219, 61-68, doi:10.1016/j.mce.2004.01.011 (2004).

\section{Acknowledgments}

This work was supported by Shanghai Committee of Science and Technology

(124119a1300; 12DZ2295005), and Renji Hospital, Shanghai JiaoTong University School of Medicine (RJZZ14-009). The funders had no role in study design, data collection and analysis, decision to publish, or preparation of the manuscript

\section{Author contributions}

Y.M., L.M.X. and H.Z.Y. performed experiments. Y.M., X.N.K. and H.L.D. conceived of the study. Y.M., Y.R.H. and X.N.K. participated in the design of this study. X.N.K., Y.R.H. and H.L.D. supervised the work. Y.M. and X.N.K. analyzed data. The manuscript was drafted by Y.M., and reviewed by all authors. All authors approved the final version of the manuscript to be published.

\section{Additional information}

Supplementary information accompanies this paper at http://www.nature.com/ scientificreports

Competing financial interests: The authors declare no competing financial interests.

How to cite this article: Ma, Y. et al. Testosterone regulates the autophagic clearance of androgen binding protein in rat Sertoli cells. Sci. Rep. 5, 8894; DOI:10.1038/srep08894 (2015).

This work is licensed under a Creative Commons Attribution 4.0 International License. The images or other third party material in this article are included in the article's Creative Commons license, unless indicated otherwise in the credit line; if the material is not included under the Creative Commons license, users will need to obtain permission from the license holder in order to reproduce the material. To view a copy of this license, visit http://creativecommons.org/licenses/by/4.0/ 